\title{
How Could Circadian Clock Genes Influence Short Duration Timing?
}

\author{
Brad Nicholas*
}

1

\author{
Introduction
}

Neuropsychiatric disorders such as autism, schizophrenia, and bipolar disorder are often associated with sleep disturbance and other circadian rhythm anomalies (Barnard and Nolan, 2008). Parsimoniously, alterations to sleep and circadian rhythms were initially considered to be accompanying problems, rather than linked with causal processes. Latterly, better understanding of the biology of circadian rhythms and the pathologies of neuropsychiatric and neurodegenerative conditions, has indicated that disregulation of circadian rhythms and sleep disturbance may critically exacerbate the development of more serious symptoms (Benca et al., 2009; Pallier et al., 2007; Thome et al., 2011).

In autism and schizophrenia, behavioural timing deficits, short duration timing anomalies and circadian rhythm anomalies, have led some researchers to consider timing deficit as an intrinsic component of these disorders (Allman, DeLeon, and Wearden, 2011; Boucher, 2001; Ward, Kellendonk, Kandel, and Balsam, 2012; Wimpory, Nicholas, and Nash, 2002). In laboratory animals (fruit fly and hamster), mutations in specific genes that regulate the circadian cycle also affect certain short period oscillations measured in seconds and minutes. This chapter explores possible mechanisms for such short period phenomena and posits the hypothesis that clock genes exert effects on neural oscillator networks supporting short period timing phenomena. This hypothesis may be relevant to research that implicates clock genes in autism, schizophrenia, and Parkinson's disease (PD).

\section{Circadian Rhythms}

The rotational planetary motion of the Earth underpins the experience of day and night, and the daily oscillation in light-intensity and temperature presents

* School of Psychology, Bangor University, Wales, UK.

(C) BRAD NICHOLAS, 2015 | DOI 10.1163/9789004230699_014 
a cycle of adversity and opportunity, a rhythm of repeating cycles of environmental challenge to life on Earth. It is perhaps hardly surprising, therefore, that cycles of about a day's length (circadian rhythms) of behaviour and physiology are observable in all organisms so far surveyed.

It has been suggested that the damaging effect of oxidative stress on cells, and particularly on the cell's genetic material (DNA), has driven selection leading to the evolution of organisms with the capacity to generate circadian rhythms in physiology and behaviour (Chen and McKnight, 2007; Edgar et al., 2012). In simple organisms such as yeast, growing in naturalistic conditions, oxidative metabolism is temporally segregated from cell division, when the DNA is particularly vulnerable to damage. Circadian rhythms of oxidative metabolism are primarily dependent on evolutionarily conserved peroxiredoxin molecules that function in a reduction-oxidation (redox) clock that generates cycles of oxidative and reductive biochemical states within the cell (Edgar et al., 2012; O'Neill and Reddy, 2011). In mammals, this redox clock is thought to mesh with a light entrainable circadian clock based on transcription factor feedback loops formed from the so-called clock genes and their protein products. Circadian rhythms can be maintained by either system alone, although their simultaneous operation appears to be required for robust cycles (Edgar et al., 2012), that are perhaps optimally advantageous.

Uchida, Hirayama, and Nishina (2010) note the presence of clock genes in signalling pathways common to the processes of cell division, the DNA damage response, and the photo-oxidative stress response triggered by ultraviolet light. They hypothesise that a cell-intrinsic yet light entrainable circadian clock would protect an organism (and particularly its genetic material) from the damaging effects of reactive oxygen species (Rоs) produced by ultraviolet light. This clock would place UV vulnerable processes like cell division, into the hours of darkness. They emphasize that this ability would have given a selective advantage to primitive organisms with such a clock that arose far back in evolutionary time.

In higher animals behaviours such as falling asleep, awakening, feeding etc., tend to occur at particular times of the day and begs the question of whether these circadian rhythms occur: i) in response to cyclic environmental stimuli or ii) due to an innate clock-like capacity that is maintained in the absence of environmental time-setting cues (zietgebers) such as light, temperature cycles, and feeding regimes. The latter case was established through investigations with model organisms such as the fruit fly Drosophila (Pittendrigh, 1966) and through human research showing that people display innate clock like properties with periods of approximately 24 hours, even when isolated from all external time cues including social cues (Aschoff, 1965; Aschoff et al., 1971). More 
recently Mills, Minors, and Waterhouse (1974) conducted a similar study to the original "Bunker" experiments of Aschoff. In this investigation, participants spent between 5 to 13 days in an isolation unit without any knowledge of time of day. Although there was some variation between participants, the majority showed cycles of physical activity and alterations in body temperature with a period of $23^{-27}$ hours, a typical range of circadian rhythm. Given that there were no environmental cues to affect these subjects, this supports innate clock-like capacity.

Neurological investigations demonstrate the primary role of small, discrete brain regions in the maintenance of circadian rhythms in animals that are the likely neurobiological loci of an innate clock like capacity. For example, the Drosophila 'clock' comprises a group of about 150 brain neurons out of a total of approximately 100,000 in the animal's central nervous system (Nitbach and Taghert, 2008). The neural network of this brain clock shows a spontaneous circadian rhythm of neural activity that is dependent on the expression of clock genes. This oscillation in these pacemaker cells is stable for weeks of constant darkness, unlike non-pacemaker cells where the oscillations damp-out in a matter of days or less. The clock is directly sensitive to light due to the presence of CRYPTOCHROME, a blue-light-sensitive protein that is present in many of the clock neurons. The ventral lateral neurons of the Drosophila brain clock may also receive neural input from the eyes of the fly, via the visual fibres (Helfrich-Forster, 2002). These two mechanisms synchronise the $24 \mathrm{~h}$ oscillation of clock gene expression in the clock neurons with the day-night cycle, and in turn, through hormone and neural output signals, the brain clock imposes a circadian rhythm on the activity patterns of the fly.

The central organismal pacemaker in mammals is the suprachiasmatic nuclei $(\mathrm{SCN})$ of the anterior hypothalamus. The phase of the self-sustaining $24 \mathrm{~h}$ cycles of neural activity in the SCN is set by neural input from the retina, to which the SCN is linked via the retinohypothalamic tract (R HT). The action of light on primarily melanopsin photoreceptors of the retina leads to a neural stimulus being transmitted along the R $\mathrm{HT}$, supported by the neurotransmitters pituitary adenylate cyclase activating peptide (PACAP) and glutamate (Berson, Dunn, and Takao, 2002; Hattar et al., 2002; Panda et al., 2002). The phase shifting properties of this stimulus on the circadian clock of the SCN depends on the subjective time that the stimulus is delivered to the SCN. Light early in the subjective night phase delays the SCN clock, while light late in the subjective night phase advances the SCN clock. The SCN exerts its influence as a masterclock by emitting neuronal and hormonal signals, in a phase-typical manner, that entrain the circadian rhythms of the peripheral organs (Balsalobre et al., 2000; Hastings, Reddy, and Maywood, 2003). 
The phase of circadian rhythms in mammals can be reset by other factors in addition to light. Feeding times (Stokkan et al., 2001), temperature changes (Brown et al., 2002), social cues (Aschoff et al., 1971; Levine, Funes, Dowse, and Hall, 2002; Mistlberger and Skene, 2004), and hormonal signals (Balsalobre et al., 2000), can each entrain or modulate circadian rhythms. However, even though the SCN shows a $24 \mathrm{~h}$ cycle of neuronal activity and output, not all the peripheral oscillators are in phase with this central clock. This is likely to be due to differences in the specific transduction pathways between SCN and each peripheral oscillator. Within the mammalian brain, for example, certain regions show circadian rhythms of activity, and the phase of one region with respect to another can vary (Feillet et al., 2008).

From the 1970s onwards, the revolution in the development of molecular biology techniques for gene manipulation, spurred investigation into the molecular and genetic aspects of circadian clocks. The period gene (per) in Drosophila melanogaster was the first example of a behavioural gene to be defined and cloned. Per is shown to regulate circadian rhythms in the fly and has become a focus of research on the mechanism and evolution of circadian clocks. The products of other so-called clock genes that interact with the per product (PERIOD protein), form a set of proteins that make up the core of the fly's circadian molecular clock. The molecular phylogeny of per and other circadian clock genes demonstrate a high degree of evolutionary conservation. Further work in mammals established that the circadian molecular clock was mechanistically similar and led to the notion of a conserved mechanism for

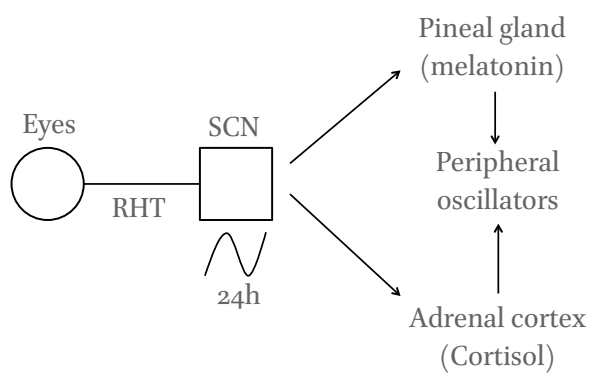

FIGURE 13.1 A schematic and simplified representation of the role of the suprachiasmatic muclei $(s c N)$ of the mammalian brain as the primary circadian pacemaker. The action of light on melanopsin containing cells of the retina can reset the phase of the $24 \mathrm{~h}$ oscillations in neural activity within the $S C N$. The $S C N$ regulates (black solid arrows) the production of certain hormones such as melatonin and cortisol that show characteristic variation in level over the day-night cycle. Cortisol and melatonin convey information of circadian phase to peripheral clocks and, thus, couple the timing of metabolic processes and behaviours to scN time. 
circadian rhythm generation amongst animals that was reflected in conserved molecular structures of clock genes and their protein products (Looby and Loudon, 2005).

3

The Circadian Molecular Clock is a System of Gene-protein Feedback Loops

The core circadian molecular clock comprises a suite of clock genes and their protein products operating as a system of transcription-translation auto-regulatory feedback loops. A heterodimer consisting of interacting proteins CLOCK and ARNTL (also known as BMAL1) is the main positive element of the circadian molecular clock and this heterodimer induces the transcription of clock controlled genes as well as the negative elements of the feedback loop (Bunger et al., 20oo; Gekakis et al., 1998). The negative elements: PER1, PER2, PER3, $\mathrm{CRY}_{1}, \mathrm{CRY}$, and NR1D1 (also known as REV-ERB $\alpha$ ), repress the action of the CLOCK/ARNTL activator and derive $\sim 24 \mathrm{~h}$ oscillations in the levels of the CLOCK/ARNTL activator via transient repression by the PER/CRY complex that is released via protein turnover and translocatory effects on the negative complex (Shearman et al., 2000).

Oscillating activity of the CLOCK/ARNTL heterodimer is coupled to rhythmic expression of clock controlled genes, induced by the binding of CLOCK/ARNTL to circadian regulatory elements (E-boxes) in the promoter regions of the clock controlled genes (Hogenesch, Gu, Jain, and Bradfield, 1998; Honma et al., 2002; Kozlov et al., 2007; Matsuo et al., 2003; Mercer et al., 2009). Second of three putative circadian regulatory elements is the D-box. Genes that contain D-Box elements are targeted by the $\mathrm{D}$ site of albumin promoter (albumin D-box) binding protein (DPB) that is a product of the clock controlled $D B P$ gene. Thus, D-Box containing genes such as RORA are second order clock controlled genes (Ueda et al., 2002, 2005). RORA and NR1D1 operate in a subsidiary circuit within the circadian clock system. These proteins regulate genes that contain a circadian regulatory element called the RevErbA/ROR binding element or RRE. RORA stimulates expression of ARNTL and CLOCK (and a homologue of $C L O C K, N P A S_{2}$ ), via RRES while $N_{1} D_{1}$ is E-box regulated and NRiDi represses ARNTL via RREs. NRiDi and RORA compete for RRE elements in the ARNTL promoter although having opposite effects on transcription; RORA acting as an enhancer and NRiDi as a repressor of the gene (Crumbley and Burris, 2011; Crumbley, Wang, Kojetin, and Burris, 2010; Preitner et al., 2002; Sato et al., 2004; Yin and Lazar, 2005; Yin, Wang, Klein, and Lazar, 2006). 
Clock controlled genes are therefore represented primarily by genes containing E-Box, D-Box, and RRE circadian regulatory elements. Some of these clock-controlled genes encode transcription factors, so that directly or indirectly at least $15 \%$ of the whole mammalian transcriptome cycles in a circadian manner (though a different set of genes may be cycling in each of the different tissues at any one time; Akhtar et al., 2002; Yang et al., 2007).

Chromatin modification plays a central role in the mechanism of transcriptional activation/repression seen in the circadian molecular clock. CLOCK is a histone acetyltransferase (Doi, Hirayama, and Sassone-Corsi, 2006) and is responsible for a circadian rhythm in histone acetylation on the promoters of PER and CRY (Etchegaray, Lee, Wade, and Reppert, 2003). The histone deacetylase SIRT1 acts upon histone $\mathrm{H}_{3}$ of the ARNTL promoter and other circadian promoters such that the circadian rhythm can be envisaged as involving circadian cycles of acetylation and de-acetylation on the promoters of clock genes (Asher et al., 2008; Nakahata et al., 2008). Cyclic methylation of histone $\mathrm{H}_{3}$ on PER and possibly $C R Y$ promoters by the polychrome protein, $\mathrm{EZH}_{2}$ is also shown to be required for the maintenance of circadian rhythm, further showing the importance of chromatin modification as intrinsic to the circadian clock mechanism (Etchegaray et al., 2006).

The negative elements of the system (PERs and $C R Y$ s) operate as a PER/CRY heterodimer that inhibits the activating effects of the CLOCK/ARNTL heterodimer. The binding of CLOCK to ARNTL facilitates binding to and repression of the CLOCK/ARNTL heterodimer by the PER/CRY complex (Hirayama et al., 2007). This complex also contains splicing factor SFPQ/PSF that recruits $\mathrm{SIN}_{3} \mathrm{~A}$ and HDACs to the $P E R$ i promoter, where deacetylation reverses the activating effect of histone acetylation by CLOCK (Duong, Robles, Knutti, and Weitz, 2011).

Eventually protein turnover, facilitated by proteins that phosphorylate, and ubiquitinylate components of the $P E R / C R Y$ complex, degrades the negative elements, and subsequent restoration of the activator complex allows the cycle to restart. Retinoic acid orphan receptor alpha, beta and gamma proteins (RORs) also facilitate by inducing expression of ARNTL, NPAS2, and CLOCK in a feed-forward circuit of the circadian molecular clock (Akashi and Takumi, 2005; Crumbley and Burris, 2011; Crumbley et al., 2010; Sato et al., 2004). The duration of migration of proteins and mRNA to and from the nucleus, respectively, together with the turnover time, broadly defines the $\sim 24 \mathrm{~h}$ period of this oscillatory system.

This core clock mechanism is coupled to the day-night cycle by genes associated with resetting the clock. Cryptochrome 1 and 2 (CRY1 and $\left.C R Y_{2}\right)$ play a critical role in matching solar time with subjective biological time by resetting 
the clock in response to light-dark cycles (Berson et al., 2002). Output pathways from the clock allow the organism to adopt a state of appropriate physiological readiness that anticipates the environmental demands associated with a particular time of day or night.

The recent discovery that neurotypical circadian clock function in mouse requires microRNAs (miR219 and miR132) adds a previously unanticipated level of complexity to the circadian molecular clock mechanism, involving clock-related gene regulation by RNA interference (Cheng et al., 2007). MIR219 is a clock-controlled microRNA gene, while $M I R r_{32}$ is light regulated and intriguingly, these microRNAs are regulators of synaptic plasticity (Impey et al., 2010). This important discovery promises new insight on the possible mechanisms behind the established effects of sleep and circadian phase on memory formation and the association of atypical circadian rhythms with neuropsychiatric disorders.

Genetic variation in clock genes is associated with neuropsychiatric disorder. For example, variation in: $C L O C K$, Attention Deficit Hyperactivity Disorder (AD HD; Kissling et al., 2008); PERt Autism (Nicholas et al., 2007); PER2, Familial Advances Sleep Phase Syndrome (Toh et al., 2001); TIMELESS, Depression (Utge et al., 2010); Seasonal Affective Disorder and Autism NPAS2 (Johansson et al., 2003; Nicholas et al., 2007) indicate involvement of clock genes in sleep, mood, and cognitive disorders (Barnard and Nolan, 2008).

Research on the biological, genetic, and molecular aspects of mammalian timing mechanisms has progressed furthest for the circadian clock with the understanding of the mechanisms sub-serving interval timing and other short period phenomena remaining underdeveloped. The close scrutiny of the circadian molecular clock has lead to findings that suggest this timer may additionally operate on scales longer and shorter than the $24 \mathrm{~h}$ period. An analogy might be made with the mechanical wristwatch or clock, where the hour hand, minute hand and second hand show different time scales but all driven from the same internal mechanism of cogs and springs. This notion of a common molecular genetic mechanisms sub-serving different biological time scales is attractive. However, to date, evidence suggests that the circadian clock only influences short period timing phenomena such as interval timing and ultradian rhythms rather than directly timing these phenomena (Beaver and Giebultowicz, 2004; Buhusi and Meck, 2005; Loudon et al., 1994). Nevertheless, it is intriguing that mutation of the clock gene period (per) in Drosophila and 
mutation of the circadian clock regulating kinase, $C S N K \imath E$ in hamster, can change the period of ultradian behaviours (Kyriacou and Hall, 1980; Loudon et al., 1994).

The following discussion moves from direct evidence for the involvement of clock genes in short period timing phenomena to considering what clock gene regulated mechanisms could support these effects. The final section of the chapter considers the relevance of clock genes to altered timing in neuropsychiatric disorders. The association of specific short period timing problems and alterations in time-sense in schizophrenia and autism for example, suggest a possible role for the circadian molecular clock in these disorders that gains some support from human genetic studies (Mansour et al., 2006; Nicholas et al., 2007).

The period gene is a particularly interesting candidate for a gene that regulates biological timing on different scales. Not only does this gene play a central role in the circadian molecular clock, as described above, but it also appears to influence the timing of developmental processes in the fruit fly, Drosophila, and temporal aspects of the male fly's "love song," a primitive form of social auditory communication. Drosophila males court females with gesture, grooming, and a courtship song. The male's song, produced by vibrating a wing, is comprised of two elements: sine song (a hum) and pulse song (a series of pulses). The rests between the pulses (the inter pulse intervals) show a cyclic modulation in length over the duration of the song. The frequency of the modulating signal ( $\mathrm{K} \& \mathrm{H}$ cycle) is $\sim 55 \mathrm{~s}$ and is regulated by the period gene (per; Alt et al., 1998; Kyriacou and Hall, 1980). Intriguingly, mutations in per that derive lengthened or shortened circadian locomotor rhythms also determine that the flies have long or short K\&H cycles, respectively. Should male flies interrupt their song, they restart singing with the K\&H cycle of the restarted song in phase with the K\&H cycle of the initial portion of the song. This suggests that the per-determined $\sim 55 \mathrm{~s} \mathrm{K \& H}$ cycle is a neuronal oscillation running as a background temporal structural element of the courtship song. Even though the fly's wings and flight muscles are primarily organs of locomotion, they also serve a special function in social communication. Correct song is a prerequisite to successful mating in Drosophila and song structure affects the receptivity of the Drosophila female (Konopka and Benzer, 1971; Konopka, Kyriacou, and Hall, 1996; Miyatake and Kanmiya, 2004; Ritchie, Halsey, and Gleason, 1999). In Bactrocera cucurbitae (melon fly), circadian mutants with short circadian locomotor rhythms also produce short pulse train intervals in the male's courtship song (Miyatake and Kanmiya, 2004). Thus, this pleiotropic effect of the circadian clock gene period on courtship song may be a characteristic of this gene. To date, as far as this author is aware, no investigations into 
the possible effects of period gene mutations on social timing systems in higher animals have been published. Nevertheless, the evolutionary conservation of the period gene suggests such studies may have interesting outcomes.

The function of period gene orthologes in timing mechanisms beyond circadian scales may be relevant to the biology and behaviour of higher animals because of the evolutionary conservation of the period gene. Drosophila per is seen to regulate developmental timing of emergence of larvae from pupa (Qiu and Hardin, 1996) and lin-42, the caenorhabidites orthologue of Drosophila period regulates the timing of molt (Monsalve, Van Buskirk, and Frand, 2011). Such definitive results are difficult to explore in Homo sapiens, nevertheless a SNP close to human PER that is associated with activity rhythms and time of death, tentatively indicates a conserved role for the period proteins in a multiscale biological timing mechanism.

Because of the characteristic interconnectedness of the individual clock elements in circadian clock function and that the circadian molecular clock mechanism is supported by there being homologues of virtually all the clock genes (PER 1, 2, and 3, CRY 1 and 2, ARNTL 1 and 2, CLOCK and NPAS 2 etc.), it is difficult to tease apart timing function that derives from a functioning clock and a specific timing phenotype that is perhaps more accurately ascribed to a particular element of the circadian clock mechanism (PER for example). Nevertheless, and for the purposes of this chapter, the question remains: How does the circadian clock or its components influence short period timing phenomena?

Studies based on pharmacological interventions implicate dopamine (Coull, Cheng, and Meck, 2011; Meck, 1996; Williamson, Cheng, Etchegaray, and Meck, 2008) and NMDA signaling in timing (Cheng, MacDonald, and Meck, 2006; Coull et al., 2011; Hata, 2011) but exactly how these signaling molecules are involved in interval timing is unclear. Timing anomalies are noted in autism (Allman, 2011), schizophrenia (Ward et al., 2012), and other neuropsychiatric disorders, such as PD, where dopamine and/or NMDA signaling is also implicated. There are points where these signaling pathways interact with the circadian clock pathway, suggesting possible avenues of influence between the circadian and interval timing mechanisms that to date remain unexplored.

The main dopamine pathways of the brain are: the nigrostriatal pathway (motor programming, attention shifting, and error prediction; Dreher and Grafman, 2002; Middleton and Strick, 2000), mesocortical pathway (working memory; 
Aalto et al., 2005; Cools et al., 2008; Kimberg, D'Esposito, and Farah, 1997), and mesolimbic pathway (motivation and reward; Pierce and Kumaresan, 2006). The dopamine antagonists, haloperidol, and remoxipride act differentially on these dopamine neural pathways and block the dopamine $\mathrm{D}_{2}$ receptor $\left(D R D_{2}\right)$. Initial experiments showed halopiridol affects all the dopamine pathways (Lidow and Goldmanrakic, 1994), while remoxipride leaves the nigrostriatal circuit unblocked (Gerlach and Casey, 1990). Rammsayer and colleagues (Rammsayer, 1993; Rammsayer and Classen, 1997) took advantage of this differential blocking in experiments with humans that implicate the nigrostriatal pathway in timing intervals of less than $500 \mathrm{~ms}$ and the mesocortical and mesolimbic pathways in timing longer intervals. The specificity of this is challenged however, by the understanding that temperature affects interval timing (Wearden and Pentonvoak, 1995) and that haloperidol affects body temperature. Nevertheless, human genetic analysis of single nucleotide polymorphisms in the dopamine $\mathrm{D}_{2}$ receptor gene $\mathrm{DRD}_{2}$ and the catechol-O-methyltransferase gene (comt, concerned with dopamine metabolism) further supports the concept of nigrostriatal dopamine pathways dealing with short duration timing and prefrontal dopamine pathways with longer duration timings (Wiener, Lohoff, and Coslett, 2011).

NMDA receptors encoded by the glutamate receptor, ionotropic, N-methyl D-aspartate genes (GRINS) are also implicated in interval timing mechanisms (Cheng et al., 2006; Coull et al., 2011; Hata, 2011) and disruption of this signaling system is implicated in schizophrenia and autism (Bangash et al., 2011; Goff and Coyle, 2001). Activation of ionotropic glutamate receptors (GRIN and GRIA genes), function in resetting the circadian clock in the SCN, a process involving phosphorylation of cAMP response element binding protein (CREB; Schurov et al., 1999) and PERı expression (Moriya, Horikawa, Akiyama, and Shibata, 2000).

PER1, a cardinal circadian clock protein that when mutated produces short duration timing effects, interacts with molecules that are implicated in NMDA and dopamine signaling. RACK1 (Receptor for Activated C Kinase 1, encoded by the gene GNB2L1) and the PER1 Interacting Protein of the SCN (PIPS) both physically interact with PERı (Hu et al., 2006; Matsuki, Kiyama, Kawabuchi, Okada, and Nagai, 2001). These two physical interactivities may implicate PER1 in short period dopamine related timing mechanisms, as pharmacological blockades to NMDA and dopamine pathways affect interval timing.

The possibilities are complex because RACKı has regulatory function in the circadian clock, dopamine signaling, and NMDAR signaling. RACK1 regulates the circadian clock by interacting with BMAL1 (Robles et al., 2010) and/or by interacting with glycogen synthase kinase 3 alpha (GSK-3a; Zeidner, Buescher, 
and Phiel, 2011). RACK1 also forms complexes with the human dopamine transporter DAT, encoded by $S C L 6 A_{3}$, and regulates phosphorylation of DAT in a Syntaxin 1a dependent mechanism. This interaction appears to modulate membrane trafficking of the dopamine transporter (Lee, Kim, Kim, and Lee, 2004). RACK1, acting as an inhibitor of phosphorylation of NM DA receptor subunits, also regulates NMDA receptor function and thereby NMDA receptormediated currents. Thus, PER1, acting as an adaptor protein, for RACK1, has the potential to modulate both NMDA and dopamine signaling pathways, possibly implicating PER1 and RACK1 in short duration timing processes via the neuromodualtory effects of glutamate and dopamine.

PIPS (the PERi interacting protein of the SCN) was discovered in the rat and this protein is homologous to the human protein GPRASP1 ( $G$ protein-coupled receptor associated sorting protein 1 ). GPRASP1 regulates the number of active D2 receptors on the cell surface by determining that the receptor, post agonist activation, is targeted to lysosomes for degradation (as opposed to endosomes for recycling; Moser et al., 2010). It is therefore plausible that PER1 may play a role in down regulating the $\mathrm{D} 2$ receptor. Consequently, misregulation of $P E R r$ might be associated either with hyper-D2R phenotypes or with hypo-D2R phenotypes, dependent on whether the PIPS-PERı interaction facilitates or impedes PIPS-D2R binding.

In addition to the specific effects of per1 on short period timing and possible mechanisms described above, the circadian clock may have a more generalized effect on timing by generating circadian oscillations in the level of molecules that influence timing. For example, genes of the dopamine signaling pathway influence short duration timing and some of these genes are circadian clock controlled.

However, there is no clear-cut overlap between the few genes known to produce interval-timing anomalies and genes that contain conserved circadian regulatory elements. Table 13.1 gives the names of genes known to affect interval timing however, none of them contain conserved circadian regulatory elements in their promoters. Neither do these genes show circadian patterns of expression in the prefrontal cortex according to Yang et al. (2007). Circadian regulation of genes of the dopamine pathway may be imposed by circadian clock regulated microRNAs targeting dopamine pathway genes. For example, conserved microRNA target sites within the 3 'UTR of the dopamine transporter gene $\mathrm{SLCFA}_{3}$ (DAT) match the seed sequences of the circadian microRNAs MIR132 and MIR219. Furthermore, circadian variation in the overall level of dopamine in the dopamine neurons of the ventral tegmental area (mouse) is reported to be due to $C L O C K$ and E-box mediated regulation of tyrosine 
hydroxylase $(T H)$, the rate-limiting enzyme in the dopamine synthesis pathway (McClung et al., 2005).

\section{Clock Genes Exert Diurnal Effects on Neuronal Architecture}

Even though the period gene influences short duration timing phenomena such as $\mathrm{K} \& \mathrm{H}$ cycles and mating durations (at least in flies; Beaver and Giebultowicz, 2004; Kyriacou and Hall, 1980), it is unlikely that the geneprotein feedback loops of the circadian molecular clock could directly derive these second-scale oscillations. Neural oscillations as emergent properties of neural networks are more likely to be the substrate for such short period phenomena. However, an explanation is still required, of how clock genes might affect neural oscillators operating in milliseconds, seconds, and minutes.

The following hypothesis attempts to draw together threads of evidence from electrophysiology and molecular genetics to derive a model that suggests how clock genes might play a role in fine-tuning the frequency of certain neural oscillator circuits. From this point of view, mutations in clock genes that influence circadian rhythms by their effect on core circadian clock genes, also have effects on clock gene regulated processes that are additional to circadian timekeeping. In this context, attention is drawn to pleiotropy (multi functionality) of certain clock genes. For example, the protein encoded by the NPAS2 gene, as well as acting as a circadian clock element, can also function as a carbon monoxide sensor protein (Dioum et al., 2002). Similarly, PER1 acts as an adaptor molecule in DNA damage response pathways additionally to its role in clock function (Sancar, Lindsey-Boltz, Unsal-Kacmaz, and Linn 2004).

TABLE 13.1 Conserved circadian control elements are not present in genes that affect interval timing.

\begin{tabular}{lll} 
Timing genes & Gene symbol & $\begin{array}{l}\text { (Conserved) } \\
\text { E-Box/D-Box/RRE }\end{array}$ \\
\hline Dopamine receptor D2 & DRD2 & no \\
Dopamine transporter (DAT) & SLC6A3 & no \\
Catechol-O-methyltransferase & COMT & no \\
Brain-specific L-proline transporter (РRОT) & $\mathrm{SLC6A}_{7}$ & no \\
& &
\end{tabular}


Drawing again on the analogy of the mechanical wristwatch, such "moonlighting" clock genes may be thought of in terms of particular cogs in a fancy watch, where the cog, as well as being essential to the timing mechanism, also drives decorative animations on the watch-face that have no direct function in telling the time.

Factors that affect the frequency of neural oscillators include the number of neurotransmitter receptors on the dendritic arbors and the degree of interarboization between the cells of the oscillator circuit. For example, Gamma $(\gamma, 20-80 \mathrm{~Hz})$ oscillations in a dendrodendritic inhibitory feedback circuit composed of mitral and granule cells of the mouse olfactory bulb occur in response to certain odors. Mutation of GABAAR- $\alpha$ subunit that alters the signal strength of the inhibitory limb of the circuit also alters the frequency of the oscillator (within limits). Computer modeling based on these and other experimental findings shows that the effect of GABAAR- $\alpha 1$ loss can be mimicked by a reduction in the number of inhibitory synapses (Lagier et al., 2007; Matsuoka, 2011). Thus, if clock genes can modulate the number of inhibitory synapses or the receptor number on the post synaptic neuron then it is plausible that level and location of circadian gene expression might influence neural oscillator frequency.

In brain clock circuits of Drosophila, rhythmic secretion of neuropeptide F causes diurnal synaptic remodeling (Fernandez et al., 2008; Fernandez, Berni, and Ceriani, 2008). Further, Berni, Beckwith, Fernandez, and Ceriani (2008) show the Drosophila gene roundabout (involved in axon guidance) also regulates activity cycles through a diurnal remodeling of neuronal branching in clock circuit cells that is down stream of and determined by the circadian molecular clock. Specifically, the clock genes per and tim regulate the size of Drosophila synaptic motor terminal boutons in a circadian manner and alter the number of neuronal projections in motor neurons in a developmental manner. Flies with lower functional tim expression show hyperbranching of neuronal projections while per mutants show hypo-branching. Thus, tim suppresses branching while per promotes branching in these Drosophila motor neurons. The double mutant (per-/tim-), however, has a normal phenotype with regards to branching (Mehnert et al., 2007; Mehnert and Cantera, 2008).

Thus, in Drosophila, there is a per/tim dependent developmental effect on a neuronal branching phenotype. It is, therefore, conceivable that differential clock gene expression in the different cell types of the brain could developmentally regulate the degree of inter-arborization of adjacent neurons of different cell types. If these were the cellular elements of a neuronal oscillator 
circuit it is possible this could affect the overall frequency of the oscillator. Thus, in principle there is precedent for clock genes and particularly the period gene, playing both a developmental and diurnal role in defining neuronal architecture. This author, therefore, proposes the hypothesis that clock genes acting as developmental and diurnal morphogenetic agents of synaptic structure and plasticity tune certain neuronal oscillator circuits. The experiments of Menhert and Cantera $(2007,2008)$ mentioned above, show how per $^{-}$flies and $\mathrm{tim}^{-}$flies show contrasting branching phenotypes but the double mutant fly does not. This intriguing observation demonstrates how alterations in the balance of expression of these clock genes and in specific tissues might be the critical factor in determining per associated short duration timing anomalies and indicates how defining phenotypes specifically linked to a given gene knock-out can be a complex process. These effects on neuronal architecture are produced by mutation of specific clock genes and this may help to explain why an experiment with Clock mutant mice did not show interval timing deficits (Cordes and Gallistel, 2008). Masking by entrainment in the interval timing experiments of Cordes and Gallistel (2008) and the regular feeding regime in the experiments by Papachristos, Jacobs, and Elgersma (2011) may possibly have obscured interval timing effects of the Clock and $\mathrm{Crys}^{-} /$ $\mathrm{Cry}_{2}{ }^{-}$mutants, respectively. Nevertheless, this masking argument notwithstanding, these mutations may not be considered to alter the ratio of CLOCK to PER.

In addition to the clock gene effects on neuronal architecture described above, the implication of the period gene in dopamine signaling pathways suggests another avenue of investigation into mechanisms whereby clock genes might regulate the frequency of neural oscillators. Dopamine supports neural network oscillations via a calcium dependent mechanism and studies of the pyloric oscillator circuit in the spiny lobster shows dopamine specifically excites or inhibits different sets of neurons in the circuit (Kloppenburg, Levini, and Harris-Warrick, 1999). This situation is analogous to the findings of Lagier et al. (2007), mentioned above, where alterations of receptor number affect excitability of specific cells with concomitant effects on oscillator frequency. Thus, altered dopamine levels can affect the frequency of neural oscillators, however, as this effect is cell type specific, the results of experiments to investigate the role of dopamine in phenotypes that involve neuronal oscillators should take account of this selectivity.

In Vivo, normal levels of dopamine in the globus pallidus and subthalamic nucleus produce gamma oscillations, $\sim 7 \mathrm{oHz}$ and reduced dopamine levels in PD in these brain regions alters the frequency of these oscillations with 
pathogenic consequences (Brown et al., 2001; Schnitzler and Gross, 2005). The implication of PER1 in dopamine signaling and altered patterns of clock gene promoter methylation and clock gene expression seen in PD may contribute to the dopamine related pathology of the disorder (Anantharam et al., 2007; Cai et al., 2010; Ding et al., 2011; Lin et al., 2012).

The concept that circadian clock proteins modulate short period timing cannot, however, be generalized for all short duration timers in an organism. Gene mutations that produce specific short duration effects appear to have specific tissues/cell types as targets. This is presumably due to the differential tissue expression-patterns of the timing genes in question. Tissue specific patterns of gene expression are maintained primarily by the interplay between DNA methylation and chromatin remodeling. The methylation of certain nucleotides in critical regions of the DNA code may be construed as an imprint upon the DNA that signals that a gene be turned "on" (un-methylated) or "off" (methylated). These methylation patterns may be specific for a particular tissue type, developmental stage, or show sex specificity. Some of these epigenetic modifications are influenced by environmental factors and some epigenetic marks can be inherited. This suggests that epigenetic regulation, that determines the expression patterns of $P E R 1, C L O C K, N P A S_{2}$ etc., may thus be as critical to oscillator function, as genetic mutations in the DNA code. In this context it is noteworthy that the primary circadian gene regulatory element, the E-box is subject to methylation at the CG dinucleotide at the centre of this feature (CACGTG). This is posited in The Social Timing Hypothesis (Wimpory, Nicholas, and Nash, 2002). A methylation screen of monozygotic twins with discordant autism diagnoses (and their non autistic siblings) highlighted differential methylation of the clock regulated gene $B C L 2$ and the clock gene RORA in the autistic twin compared with the co-twin and unaffected sib. Further immunohistochemical analysis of autism brain tissue showed altered expression of BCL2 and RORA compared to controls and significant genomewide epigenetic parent-of-origin effects for autism at the CLOCK locus tentatively implicate clock gene methylation in the disorder (Fradin et al., 2010; Nguyen, Rauch, Pfeifer, and Hu, 2010). A study of clock gene promoter methylation in mouse, found major changes in clock gene E-Box region methylation around the perinatal period of development, suggesting a role for developmentally programmed methylation of clock gene regulatory elements that could contribute to tissue specificity of clock gene expression patterns (Ji, Qin, $\mathrm{Shu}$, and $\mathrm{Li}, 2010$ ). This is in keeping with a role for clock gene methylation in later developmental programmes indicated by altered methylation of clock genes in obesity, cancer, and PD (Chen et al., 2005; Lin et al., 2012; Milagro et al., 2012). 
Altered sleep patterns and circadian rhythm anomalies accompany many neuropsychiatric, neurodevelopmental, and neurodegenerative disorders and this disruption contributes to the severity and/or development of these disorders. The process by which circadian disregulation contributes to these disorders is however not extensively explored. The neuronal architecture of brain oscillators and neural networks is maintained within strict limits, under homeostatic control and some of the genes that regulate this homeostatic process are clockcontrolled genes. It is, therefore, plausible that this interaction between the homeostatic and circadian process may lead to homeostatic failure if the circadian signal is attenuated or boosted beyond a certain limit.

Ramocki and Zoghbi (2008) drawing on findings that certain gene copy number variants are associated with autism, schizophrenia, and Rett syndrome, stress that duplication or deletion of certain critical genes can give rise to remarkably similar neuropsychiatric phenotypes. Further, analysis of altered neuronal architecture that is a consequence of some of these deletions and duplications show hyper- and hypo-branching morphologies. From this, Ramocki and Zoghbi conclude that healthy neurons must maintain within limits, the number and structure of synapses and certain genes are critical in this respect, $M E C P_{2}$ for example. They propose that failure of neuronal homeostasis is the basis of autism as a feature of several neurodevelopmental disorders. Neuronal homeostatic processes ensure normal synaptic function by maintaining neuronal architecture and excitability within certain critical and neurotypical limits. Genes that need to be tightly regulated to maintain neuronal homeostasis may also be clock controlled genes. If so, then mutations that alter the amplitude or location of the circadian signal could boost (or fail to boost) neuropsychiatric relevant genes beyond their normal working limits with pathological consequences.

The main circadian regulatory element, the gene promoter E-Box, is primarily an enhancer element. E-box binding by the cardinal positive element of the circadian molecular clock (CLOCK- or NPAS2-/ARNTL heterodimer), boosts transcription over the baseline constitutive level. A number of genes associated with neuropsychiatric disorders are known to contain at least one conserved E-Box in their promoter regions and to show circadian patterns of expression in brain tissues (Yang et al., 2007).

In this context, it is plausible that the influence of gene/epigenetic variants that alter constitutive levels of clock controlled gene expression, would be additive to variants of clock genes specifically, that affect the amplitude etc. of circadian cycles. Further, the dopamine receptor DRD2 potentiates CLOCK/ 

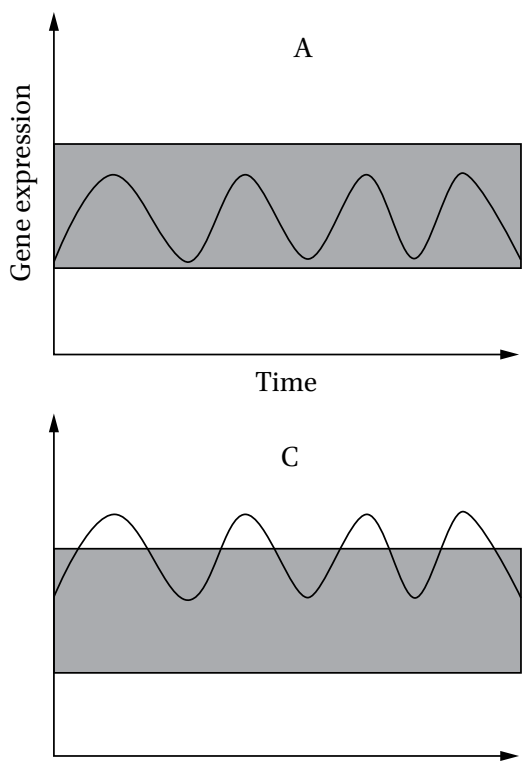
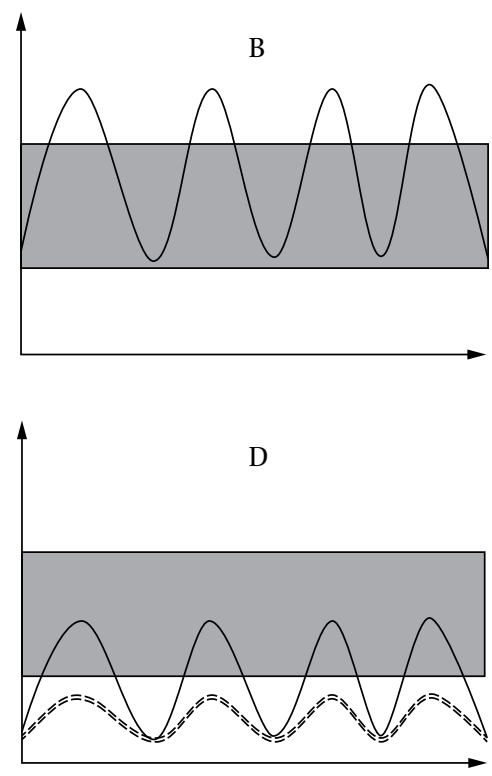

FIGURE 13.2 A schematic representation of the interaction between Neuronal Homeostatic Limit (grey box) of expression of neuronal genes and circadian regulation of one of these neuronal genes (sinusoid); graphs A, B, and C. A, neurotypical; $B$, variation causing a circadian signal with increased amplitude; $C$, variation causing increased base line (constitutive) expression of clock controlled gene. D represents another clock-controlled gene where neurotypical circadian boosting (solid line)fails due to mutation that produces a week circadian signal (dashes).

BMAL1 activation of E-boxes (Yujnovsky, Hirayama, Doi, Borrelli, and SassoneCorsi, 2006), suggesting variation in the dopamine pathway could increase/ decrease the amplitude of cycles of clock controlled gene expression.

It is clear that clock genes and the circadian clock regulate the expression levels of certain genes relevant to development and maintenance of neuronal architecture and excitability. Figure 13.2 describes three scenarios where anomalous expression of clock genes could cause the expression levels of clock-controlled genes to fall outside the normal limits of neuronal homeostasis. This hypothesis, of circadian effects on homeostatic limits, shows how genetic variation at a multitude of different points in the system could lead to the same effect, failure of homeostasis that is driven by the action of the circadian clock. In Figure 13.1, four scenarios are presented: A-neurotypical, B-high amplitude clock signal, C-normal clock signal plus increased constitutive expression level of clock controlled genes, and D-decreased amplitude of clock signal. Scenario D could pertain to situations such as circadian effects on 
memory consolidation where a circadian boost is required at a specific time point, during sleep for example, and where inadequate boosting would lead to inadequate expression to support consolidation. Under such scenarios, pinning down genetic effects in neuropsychiatric disorders such as autism, and at the level of populations, could be exceedingly complex, as appears to be the case. Examining DNA sequence variation alone would be inadequate, and risk could be spread thinly, over a large number of variants of small effect. Epigenetic mis-regulation of neuronal genes and clock genes (known to occur in cancer; Gery et al., 2007) and which dictates tissue expression patterns, would need to be taken into consideration. Thus, epigenetic and genetic effects, within clock genes or in clock-controlled genes could be pathologically additive. On the other hand, the involvement of epigenetic effects and common association of circadian disruption in neuropsychiatric disorders suggests possible avenues of treatment. Epigenetic effects are currently potentially more amenable to pharmacological reprogramming compared to DNA variations and circadian problems are already being addressed with drugs such as lithium and melatonin. As more personalised genomics become possible, there is growing potential for chrono-directed drug administration and chronotherapies that takes account of individual genetic differences in the circadian clock and the systems it regulates.

\section{8}

\section{Summary}

Circadian rhythms in physiology and behaviour are endogenous biological cycles of $\sim 24 \mathrm{~h}$ reliant on the cyclic expression of genes, the so-called clock genes, that encode transcription factors. Circadian rhythm disruption is apparent in disorders such as autism, schizophrenia, and bipolar disorder and genetic association studies indicate that variations in clock genes and/or clock related genes may contribute to the circadian disturbances in these disorders.

Experimental evidence supports the notion that clock genes may influence short period timing by an effect on neural oscillator networks mediated by dopamine signaling pathways or alterations in neuronal architecture or excitability. Association of short duration timing anomalies and sleep and circadian rhythm disturbance with schizophrenia and autism suggest these disturbances may possibly both derive from or be contributed to, by circadian molecular clock anomalies.

Psychologists have pondered the developmental effects of infants growing up with timing difficulties, especially a short duration timing deficit that affects 
processing speed during social interaction (see Chapter 3 of this book), and the cognitive impact of sleep disturbance is well known. Clearly disturbed sleep and circadian rhythms cannot of themselves cause autism in children or schizophrenia in adults, but the possibility that a timing deficit contributes to autism and schizophrenia is plausible. Clinically, the amenability of the circadian clock to treatments with low risk pharmaceuticals and the availability of behavioural interventions that structure and support timing in social interaction encourage further exploration.

\section{References}

Aalto, S. et al. 2005. "Frontal and Temporal Dopamine Release during Working Memory and Attention Tasks in Healthy Humans: A Positron Emission Tomography Study Using the High-Affinity Dopamine D-2 Receptor Ligand C-11 FLB 457" Journal of Neuroscience 25(10): 2471-7.

Akashi, M., and T. Takumi. 2005. "The Orphan Nuclear Receptor ROR Alpha Regulates Circadian Transcription of the Mammalian Core-Clock Bmalı" Nature Structural and Molecular Biology 12(5): 441-8.

Akhtar, R.A. et al. 2002. "Circadian Cycling of the Mouse Liver Transcriptome, as Revealed by cDNA Microarray, is Driven by the Suprachiasmatic Nucleus" Current Biology 12(7): 540-50.

Allman, M.J. 2011. "Deficits in Temporal Processing Associated with Autistic Disorder" Frontiers in Integrative Neuroscience 5.

Allman, M.J., I.G. DeLeon, and J.H. Wearden. 2011. "Psychophysical Assessment of Timing in Individuals with Autism" American Journal on Intellectual and Developmental Disabilities 116(2): 165-78.

Alt, S. et al. 1998. "The Period Gene Controls Courtship Song Cycles in Drosophila Melanogaster" Animal Behaviour 56: 87-97.

Anantharam, V. et al. 2007. "Microarray Analysis of Oxidative Stress Regulated Genes in Mesencephalic Dopaminergic Neuronal Cells: Relevance to Oxidation Damage in Parkinson's Disease" Neurochemistry International 5o(6): 834-47.

Aschoff, J.1965. "Circadian Rhythms in Man - A Self-Sustained Oscillator with an Inherent Frequency Underlies Human 24-Hour Periodicity" Science 148(3676): 1427-32.

Aschoff, J. et al. 1971. "Human Circadian Rhythms in Continuous Darkness Entrainment by Social Cues" Science 171(3967): 213-5.

Asher, G. et al. 2008. "SIRT1 Regulates Circadian Clock Gene Expression through PER2 Deacetylation." Cell 134(2): 317-28.

Balsalobre, A. et al. 200o. "Resetting of Circadian Time Peripheral Tissues by Glucocorticoid Signaling" Science 289(5488): 2344-7. 
Bangash, Park, Tatiana Melnikova et al. 2011. "Enhanced Polyubiquitination of Shank3 and NMDA Receptor in a Mouse Model of Autism" Cell 145(5): 758-72.

Barnard A.R., and P.M. Nolan. 2008. "When Clocks Go Bad: Neurobehavioural Consequences of Disrupted Circadian Timing” PLoS Genetics. 4(5)e1000040.

Beaver, L.M., and J.M. Giebultowicz. 2004. "Regulation of Copulation Duration by Period and Timeless in Drosophila Melanogaster" Current Biology 14(16): 1492-7.

Benca R. et al. 2009. "Biological Rhythms, Higher Brain Function, and Behavior: Gaps, Opportunities, and Challenges" Brain Research Reviews 62(1): 57-70.

Berni, J. et al. 2008. "The Axon-Guidance Roundabout Gene Alters the Pace of the Drosophila Circadian Clock" European Journal of Neuroscience 27(2): 396-407.

Berson, D.M., F.A. Dunn, and M. Takao. 2002. "Phototransduction by Retinal Ganglion Cells that Set the Circadian Clock" Science 295(5557): 1070-3.

Boucher, J. 2001. "Lost in a Sea of Time': Time Parsing and Autism” In C. Hoerl, and T. Cormack (Eds.), Time and Memory. Oxford: Clarendon.

Brown, P. et al. 2001. "Dopamine Dependency of Oscillations between Subthalamic Nucleus and Pallidum in Parkinson's Disease” Journal of Neuroscience 21(3): 1033-8. Brown, S.A. et al. 2002. "Rhythms of Mammalian Body Temperature Can Sustain Peripheral Circadian Clocks" Current Biology 12(18): 1574-83.

Buhusi, C.V., and W.H. Meck. 2005. "What Makes Us Tick? Functional and Neural Mechanisms of Interval Timing" Nature Reviews Neuroscience 6(10): 755-65.

Bunger, M.K. et al. 2000. "Mop3 is an Essential Component of the Master Circadian Pacemaker in Mammals" Cell 103(7): 1009-17.

Cai, Y. et al. 2010. "Expression of Clock Genes Perı and Bmalı in Total Leukocytes in Health and Parkinson's Disease" European Journal of Neurology 4: 550-4.

Chen, S.T. et al. 2005. "Deregulated Expression of the PER1, PER2 and PER3 Genes in Breast Cancers" Carcinogenesis 26(7): 1241-6.

Chen, Z., and S.L. McKnight. 2007. "A Conserved DNA Damage Response Pathway Responsible for Coupling the Cell Division Cycle to the Circadian and Metabolic Cycles" Cell Cycle 6(23): 2906-12.

Cheng, H.Y.M. et al. 2007. "microRNA Modulation of Circadian-Clock Period and Entrainment" Neuron 54(5): 813-29.

Cheng, R.-K., C.J. MacDonald, and W.H. Meck. 2006. "Differential Effects of Cocaine and Ketamine on Time Estimation: Implications for Neurobiological Models of Interval Timing" Pharmacology Biochemistry and Behavior 85(1): 114-22.

Cools, R. et al. 2008. “Working Memory Capacity Predicts Dopamine Synthesis Capacity in the Human Striatum" Journal of Neuroscience 28(5): 1208-12.

Cordes, S., and C.R. Gallistel. 2008. "Intact Interval Timing in Circadian CLOCK mutants" Brain Research, 1227.

Coull, J.T., R.-K. Cheng, and W.H. Meck. 2011. "Neuroanatomical and Neurochemical Substrates of Timing" Neuropsychopharmacology 36(1): $3^{-25}$. 
Crumbley, C., and T.P. Burris. 2011. "Direct Regulation of CLOCK Expression by REVERB” Plos One 6(3): e1729o.

Crumbley, C. et al. 2010. "Characterization of the Core Mammalian Clock Component, NPAS2, as a REV-ERB alpha/ROR Alpha Target Gene” Journal of Biological Chemistry 285(46): 35386-92.

Ding, H. et al. 2011. "Decreased Expression of Bmal2 in Patients with Parkinson's Disease" Neuroscience Letters 499(3): 186-8.

Dioum, E.M. et al. 2002. "NPAS2: A Gas-Responsive Transcription Factor" Science 298(5602): 2385-7.

Doi, M., J. Hirayama, and P. Sassone-Corsi. 2006. "Circadian Regulator CLOCK is a Histone Acetyltransferase" Cell 125(3): 497-508.

Dreher, J.C., and J. Grafman. 2002. "The Roles of the Cerebellum and Basal Ganglia in Timing and Error Prediction" European Journal of Neuroscience 16(8): 1609-19.

Duong, H.A. et al. 2011. "A Molecular Mechanism for Circadian Clock Negative Feedback" Science 332(6036): 1436-9.

Edgar, R.S. et al. 2012. "Peroxiredoxins are Conserved Markers of Circadian Rhythms" Nature 485(7399): 459-64.

Etchegaray, J.P. et al. 2003. "Rhythmic Histone Acetylation Underlies Transcription in the Mammalian Circadian Clock" Nature 421(6919): 177-82.

Etchegaray, J.P. et al. 2006. "The Polycomb Group Protein EZH2 is Required for Mammalian Circadian Clock Function" Journal of Biological Chemistry 281(30): 21209-15.

Feillet, C.A. et al. 2008. "Forebrain Oscillators Ticking with Different Clock Hands" Molecular and Cellular Neuroscience 37(2): 209-21.

Fernandez, M.P., J. Berni, and M.F. Ceriani. 2008. "Circadian Remodeling of Neuronal Circuits Involved in Rhythmic Behavior" Plos Biology 6(3): 518-24.

Fradin, D. et al. 2010. "Parent-of-Origin Effects in Autism Identified through GenomeWide Linkage Analysis of 16,00o SNPs" Plos One 5(9): e12513.

Gekakis, N. et al. 1998. "Role of the CLOCK Protein in the Mammalian Circadian Mechanism" Science 280(5369): 1564-9.

Gerlach, J., and D.E. Casey. 1990. "Remoxipride, a New Sellective D2 Antagonist, and Haloperidol in Cebus Monkeys" Progress in Neuro-Psychopharmacology and Biological Psychiatry 14(1): 103-12.

Gery, S. et al. 2007. "Epigenetic Silencing of the Candidate Tumor Suppressor Gene Per1 in Non-Small Cell Lung Cancer" Clinical Cancer Research 13(5): 1399-404.

Goff, D.C., and J.T. Coyle. 2001 "The Emerging Role of Glutamate in the Pathophysiology and Treatment of Schizophrenia" Ammerican Journal of Psychiatry 158(9): 1367-77.

Hastings, M.H., A.B. Reddy, and E.S. Maywood. 2003. "A Clockwork Web: Circadian Timing in Brain and Periphery, in Health and Disease" Nature Reviews Neuroscience 4(8): 649-61.

Hata, T. 2011. "Glutamate - A Forgotten Target for Interval Timing" Frontiers in Integrative Neuroscience 5: 27. 
Hattar, S. et al. 2002. "Melanopsin-Containing Retinal Ganglion Cells: Architecture, Projections, and Intrinsic Photosensitivity" Science 295(5557): 1065-70.

Hayes, K.R., J.E. Baggs, and J.B. Hogenesch. 2005. "Circadian Clocks are Seeing the Systems Biology Light" Genome Biology 6(5): 219.

Helfrich-Förster, C. 2002. "The Circadian System of Drosophila Melanogaster and Its Light Input Pathways" Zoology 105(4): 297-312.

Hirayama, J. et al. 2007. "CLOCK-Mediated Acetylation of BMALı Controls Circadian Function" Nature 450: 1086-90.

Hogenesch, J.B. et al. 1998. "The Basic-Helix-Loop-Helix-PAS Orphan MOP3 Forms Transcriptionally Active Complexes with Circadian and Hypoxia Factors" Proceedings of the National Academy of Sciences of the United States of America 95(10): 5474-9.

Honma, S. et al. 2002. "Dec1 and Dec2 are Regulators of the Mammalian Molecular Clock" Nature 419(6909): 841-4.

$\mathrm{Hu}$, L.J. et al. 2006. "RACK1, A Novel hPER1-Interacting Protein" Journal of Molecular Neuroscience 29(1):55-63.

Impey, S. et al. 2010. "An Activity-Induced microRNA Controls Dendritic Spine Formation by Regulating Rac1-PAK Signaling" Molecular and Cellular Neuroscience $43(1): 146-56$.

Ji, Y. et al. 2010. "Methylation Analyses on Promoters of mPer1, mPer2, and mCryı

During Perinatal Development" Biochemical and Biophysical Research Communications 391(4): 1742-7.

Johansson C. et al. 2003. "Circadian Clock-Related Polymorphisms in Seasonal Affective Disorder and Their Relevance to Diurnal Preference" Neuropsychopharmacology 28(4): 734-9.

Kimberg, D.Y., M. D’Esposito, and M.J. Farah. 1997. "Cognitive Functions in the Prefrontal Cortex - Working Memory and Executive Control" Current Directions in Psychological Science 6(6): 185-92.

Kissling C. et al. 2008. "A Polymorphism at the 3'-Untranslated Region of the CLOCK Gene is Associated with Adult Attention-Deficit Hyperactivity Disorder" American Journal of Medical Genetics B Neuropsychiatric Genetics 147(3): 333-8.

Kloppenburg, P., R.M. Levini, and R.M. Harris-Warrick. 1999. “Dopamine Modulates Two Potassium Currents and Inhibits the Intrinsic Firing Properties of an Identified Motor Neuron in a Central Pattern Generator Network" Journal of Neurophysiology 81(1).

Konopka, R.J., and S. Benzer. 1971. "Clock Mutants of Drosophila-Melanogaster" Proceedings of the National Academy of Sciences of the United States of America 68(9): 2112-6.

Konopka, R.J., C.P. Kyriacou, and J.C. Hall. 1996. "Mosaic Analysis in the Drosophila CNS of Circadian and Courtship-Song Rhythms Affected by a Period Clock Mutation" Journal of Neurogenetics 11(1-2): 117-39.

Kozlov, S.V. et al. 2007. "The Imprinted Gene Magel2 Regulates Normal Circadian Output" Nature Genetics 39(10): 1266-72. 
Kyriacou, C.P., and J.C. Hall. 1980. "Circadian-Rhythm Mutations in DrosophilaMelanogaster Affect Short-Term Fluctuations in the Males Courtship Song" Proceedings of the National Academy of Sciences of the United States of AmericaBiological Sciences 77(11): 6729-33.

Lagier, S. et al. 2007. "GABAergic Inhibition at Dendrodendritic Synapses Tunes Gamma Oscillations in the Olfactory Bulb" Proceedings of the National Academy of Sciences of the United States of America 104(17): 7259-64.

Lee, K.H. et al. 2004. "Syntaxin $1 A$ and Receptor for Activated C Kinase Interact with the N-Terminal Region of Human Dopamine Transporter" Neurochemical Research 29(7): $1405^{-9}$.

Levine, J.D. et al. 2002. "Resetting the Circadian Clock by Social Experience in Drosophila Melanogaster" Science 298(5600): 2010-2.

Lidow, M.S., and P.S. Goldmanrakic. 1994. "A Common Action of Clozapine, Haloperidol, and Remoxipride on D-1-Dopaminergic and D-2-Dopaminergic Receptors in the Primate Cerebral-Cortex" Proceedings of the National Academy of Sciences of the United States of America 91(10): 4353-6.

Lin, Q. et al. 2012. "Promoter Methylation Analysis of Seven Clock Genes in Parkinson's Disease" Neuroscience Letters 507(2): 147-50.

Looby, P., and A.S.I. Loudon. 2005. "Gene Duplication and Complex Circadian Clocks in Mammals" Trends in Genetics 21(1): 46-53.

Loudon, A.S.I. et al. 1994. "Ultradian Endocrine Rhythms are Altered by a Circadian Mutation in the Syrian-Hamster" Endocrinology 135(2): 712-8.

Mansour, H.A. et al. 2006. "Association Study of Eight Circadian Genes with Bipolar I Disorder, Schizoaffective Disorder and Schizophrenia" Genes Brain and Behavior $5(2): 150-7$.

Matsuki, T. et al. 2001. "A Novel Protein Interacts with a Clock-Related Protein, rPerı" Brain Research 916(1-2): 1-10.

Matsuo, T. et al. 2003. "Control Mechanism of the Circadian Clock for Timing of Cell Division in Vivo" Science 302(5643): 255-9.

Matsuoka, K. 2011. "Analysis of a Neural Oscillator" Biological Cybernetics 104(4-5): 297-304.

McClung, C.A. et al. 2005. "Regulation of Dopaminergic Transmission and Cocaine Reward by the Clock Gene" Proceedings of the National Academy of Sciences of the United States of America 102(26): 9377-81.

Meck, W.H. 1996. "Neuropharmacology of Timing and Time Perception" Brain Research. Cognitive Brain Research 3(3-4): 227-42.

Mehnert, K.I. et al. 2007. "Circadian Changes in Drosophila Motor Terminals" Developmental Neurobiology 67(4): 415-21.

Mehnert, K.I., and R. Cantera. 2008. "A Peripheral Pacemaker Drives the Circadian Rhythm of Synaptic Boutons in Drosophila Independently of Synaptic Activity" Cell and Tissue Research 334(1): 103-9. 
Mercer, R.E. et al. 2009. "Regionally Reduced Brain Volume, Altered Serotonin Neurochemistry, and Abnormal Behavior in Mice Null for the Circadian Rhythm Output Gene Magel2" American Journal of Medical Genetics Part B-Neuropsychiatric Genetics $150 \mathrm{oB}(8)$ : 1085-99.

Middleton, F.A., and P.L. Strick. 200o. "Basal Ganglia Output and Cognition: Evidence from Anatomical, Behavioral, and Clinical Studies" Brain and Cognition 42(2): 183-200.

Milagro, F.I. et al. 2012. "CLOCK, PER2 and BMAL1 DNA Methylation: Association with Obesity and Metabolic Syndrome Characteristics and Monounsaturated Fat Intake" Chronobiology International 29(9): 1180-94.

Mistlberger, R.E., and D.J. Skene. 2004. "Social Influences on Mammalian Circadian Rhythms: Animal and Human Studies" Biological Reviews 79(3): 533-56.

Miyatake, T., and K. Kanmiya. 2004. "Male Courtship Song in Circadian Rhythm Mutants of Bactrocera Cucurbitae(Tephritidae:Diptera)" Journal of Insect Physiology 50(1): 85-91.

Monsalve, G.C., C. Van Buskirk, and A.R. Frand. 2011. "LIN-42/PERIOD Controls Cyclical and Developmental Progression of C-elegans Molts" Current Biology 21(24): 2033-45.

Moriya, T. et al. 2000. "Correlative Association between N-methyl-D-aspartate ReceptorMediated Expression of Period Genes in the Suprachiasmatic Nucleus and Phase Shifts in Behavior with Photic Entrainment of Clock in Hamsters" Molecular Pharmacology 58(6): 1554-62.

Moser, E. et al. 2010. "G Protein-Coupled Receptor-Associated Sorting Protein 1 Regulates the Postendocytic Sorting of Seven-Transmembrane-Spanning G ProteinCoupled Receptors" Pharmacology 86(1): 22-9.

Nakahata, Y. et al. 2008. "The NAD(+)-dependent Deacetylase SIRT1 Modulates CLOCKMediated Chromatin Remodeling and Circadian Control" Cell 134(2): 329-40.

Nguyen, A. et al. 2010. "Global Methylation Profiling of Lymphoblastoid Cell Lines Reveals Epigenetic Contributions to Autism Spectrum Disorders and a Novel Autism Candidate Gene, RORA, Whose Protein Product is Reduced in Autistic Brain" Faseb Journal 24(8): 3036-51.

Nicholas, B. et al. 2007. "Association of Per1 and Npas2 with Autistic Disorder: Support for the Clock Genes/Social Timing Hypothesis" Molecular Psychiatry 12(6): 581-92.

Nitabach, M.N., and P.H. Taghert. 2008. "Organization of the Drosophila Circadian Control Circuit" Current Biology 18(2): R84-93.

O'Neill, J.S., and A.B. Reddy. 2011. "Circadian Clocks in Human Red Blood Cells" Nature 469(7331): 498-503.

Pallier, P.N. et al. 2007. "Pharmacological Imposition of Sleep Slows Cognitive Decline and Reverses Dysregulation of Circadian Gene Expression in a Transgenic Mouse Model of Huntington's Disease" Journal of Neuroscience 27(29): 7869-78.

Panda, S. et al. 2002. "Melanopsin (Opn4) Requirement for Normal Light-Induced Circadian Phase Shifting" Science 298(5601): 2213-6. 
Papachristos, E.B., E.H. Jacobs, and Y. Elgersma. 2011. "Interval Timing Is Intact in Arrhythmic Cry1/Cry2-Deficient Mice" Journal of Biological Rhythms 26(4): 305-13.

Pierce, R.C., and V. Kumaresan. 2006. "The Mesolimbic Dopamine System: The Final Common Pathway for the Reinforcing Effect of Drugs of Abuse?" Neuroscience and Biobehavioral Reviews 30(2): 215-38.

Pittendrigh, C.S. 1966. "The Circadian Oscillation in Drosophila Pseudoobscura Pupae: A Model for the Photoperiodic Clock" Z Pflanzenphysiol Z Bot 54(4): 275-307.

Preitner, N. et al. 2002. "The Orphan Nuclear Receptor REV-ERB Alpha Controls Circadian Transcription within the Positive Limb of the Mammalian Circadian Oscillator" Cell 110(2): 251-6o.

Qiu, J., and P.E. Hardin. 1996. "Developmental State and the Circadian Clock Interact to Influence the Timing of Eclosion in Drosophila Melanogaster" Journal of Biological Rhythms 11(1): 75-86.

Rammsayer, T.H. 1993. "On Dopaminergic Modulation of Temporal InformationProcessing" Biological Psychology 36(3): 209-22.

Rammsayer, T., and W. Classen. 1997. “Impaired Temporal Discrimination in Parkinson's Disease: Temporal Processing of Brief Durations as an Indicator of Degeneration of Dopaminergic Neurons in the Basal Ganglia" International Journal of Neuroscience 91(1-2): 45-55.

Ramocki, M.B., and H.Y. Zoghbi. 2008. "Failure of Neuronal Homeostasis Results in Common Neuropsychiatric Phenotypes" Nature 455(7215): 912-8.

Ritchie, M.G., E.J. Halsey, and J.M. Gleason. 1999. "Drosophila Song as a Species-Specific Mating Signal and the Behavioural Importance of Kyriacou and Hall Cycles in D-Melanogaster Song" Animal Behaviour 58: 649-57.

Robles, M.S. et al. 2010. "Identification of RACK1 and Protein Kinase C Alpha as Integral Components of the Mammalian Circadian Clock" Science 327(5964): 463-6.

Sancar, A. et al. 2004. "Molecular Mechanisms of Mammalian DNA Repair and the DNA Damage Checkpoints" Annual Review of Biochemistry 73: 39-85.

Sato, T.K. et al. 2004. "A Functional Genomics Strategy Reveals Rora as a Component of the Mammalian Circadian Clock" Neuron 43(4): 527-37.

Schnitzler, A., and J. Gross. 2005. "Normal and Pathological Oscillatory Communication in the Brain" Nature Reviews Neuroscience 6(4): 285-96.

Schurov, I.L. et al. 1999. "Glutamatergic Induction of CREB Phosphorylation and Fos Expression in Primary Cultures of the Suprachiasmatic Hypothalamus in Vitro is Mediated by Co-ordinate Activity of NMDA and Non-NMDA Receptors" Journal of Neuroendocrinology 11(1): 43-51.

Shearman, L.P. et al. 200o. "Targeted Disruption of the mPer3 Gene: Subtle Effects on Circadian Clock Function" Molecular and Cellular Biology 20(17): 6269-75.

Stokkan, K.A. et al. 2001. "Entrainment of the Circadian Clock in the Liver by Feeding" Science 291(5503): 490-3. 
Thome, J. et al. 2011. "Clock Genes and Circadian Rhythmicity in Alzheimer Disease" Aging Research 2011: 383091.

Toh, K.L. et al. 2001. "An hPer2 Phosphorylation Site Mutation in Familiar Advanced Sleep Phase Syndrome" Science 291(5506): 1040-3.

Uchida, Y., J. Hirayama, and H. Nishina. 2010. "A Common Origin: Signaling Similarities in the Regulation of the Circadian Clock and DNA Damage Responses" Biological and Pharmaceutical Bulletin 33(4): 535-44.

Utge, S.J. et al. 2010. "Systematic Analysis of Circadian Genes in a Population-Based Sample Reveals Association of TIMELESS with Depression and Sleep Disturbance" Plos One 5(2): eg259.

Ueda, H.R. et al. 2002. "A Transcription Factor Response Element for Gene Expression During Circadian Night" Nature 418(6897): 534-9.

Ueda, H.R. et al. 2005. "System-Level Identification of Transcriptional Circuits Underlying Mammalian Circadian Clocks" Nature Genetics 37(2): 187-92.

Ward, R.D. et al. 2012. "Timing as a Window on Cognition in Schizophrenia" Neuropharmacology 62(3): 1175-81.

Wearden, J.H., and I.S. Pentonvoak. 1995. "Feeling the Heat - Body-Temperature and the Rate of Subjective Time, Revisited" Quarterly Journal of Experimental Psychology Section B-Comparative and Physiological Psychology 48(2): 129-41.

Wiener, M., F.W. Lohoff, and H.B. Coslett. 2011. "Double Dissociation of Dopamine Genes and Timing in Humans" Journal of Cognitive Neuroscience 23(10): 2811-21.

Williamson, L.L. et al. 2008. 'Speed' Warps Time: Methamphetamine's Interactive Roles in Drug Abuse, Habit Formation, and the Biological Clocks of Circadian and Interval Timing" Current Drug Abuse Reviews 1(2): 203-12.

Wimpory, D., B. Nicholas, and S. Nash. 2002. "Social Timing, Clock Genes and Autism: A New Hypothesis" Journal of Intellectual Disability Research 46: 352-8.

Yang, S.Z. et al. 2007. "Genome-Wide Expression Profiling and Bioinformatics Analysis of Diurnally Regulated Genes in the Mouse Prefrontal Cortex" Genome Biology 8(11): R247. Yin, L., and M.A. Lazar. 2005. "The Orphan Nuclear Receptor Rev-erb Alpha Recruits the N-CoR/histone Deacetylase 3 Corepressor to Regulate the Circadian Bmalı Gene" Molecular Endocrinology 19(6): 1452-9.

Yin, L. et al. 2006. "Nuclear Receptor Rev-erba is a Critical Lithium-Sensitive Component of the Circadian Clock" Science 311(5763): 1002-5.

Yujnovsky, I. et al. 2006. "Signaling Mediated by the Dopamine D2 Receptor Potentiates Circadian Regulation by CLOCK:BMAL1" Proceedings of the National Academy of Sciences of the United States of America 103(16): 6386-91.

Zeidner, L.C., J.L. Buescher, and C.J. Phiel. 2011. "A Novel Interaction between Glycogen Synthase Kinase-3alpha (GSK-3alpha) and the Scaffold Protein Receptor for Activated C-Kinase 1 (RACK1) Regulates the Circadian Clock" International Journal of Biochemistry and Molecular Biology 2(4): 318-27. 